\title{
Utilização da goma metilcelulose para redução da absorção de gordura em produtos empanados
}

\author{
Use of the methylcellulose gum for fat up-take absorption \\ in coated products
}

\author{
Marcia de Mello Luvielmo ${ }^{1}$; Daniele Domingues Dill ${ }^{2}$
}

\section{Resumo}

O empanamento de carnes, apesar de agregar valor aos produtos e conquistar a confiança do consumidor, melhorando aparência e sabor, possui uma etapa de pré-fritura, durante o processamento, que propicia a saída de água e entrada de gordura no produto, aumentando, com isso, o teor de gordura do produto. A proposta deste trabalho foi desenvolver um sistema de empanamento que absorva menos gordura durante a etapa de pré-fritura de um produto empanado de frango. Para isso, foi utilizada a adição de metilcelulose (MC) em diferentes concentrações nos sistemas de cobertura, batter llíquido de empanamento) e breading (cobertura externa). Foram realizados cinco experimentos, um experimento padrão, sem a adição de ingrediente com função tecnológica, formulações com adição de $\mathrm{MC}$ no batter ( $0,5 \%$ e $2,0 \%)$, com adição de MC no batter e no breading ( $1 \%$ em cada) e com adição de MC no breading (2,0\%). Os resultados encontrados para a redução na absorção de gordura nas formulações com 0,5 e 2,0\% de MC no batter foram de 5,81\% e 8,40\%, respectivamente, para o experimento que recebeu $1 \%$ de $\mathrm{MC}$ no batter e $1 \%$ de MC no breading,, a redução de gordura foi de 7,66\%. No experimento que recebeu $2 \%$ de MC no breading a redução de gordura foi de $10,51 \%$. As formulações com $2 \%$ de MC adicionada ao breading (10,51\%) e $2 \%$ de MC adicionada ao batter $(8,40 \%)$ obtiveram as maiores reduções de absorção de gordura durante a etapa de pré-fritura. No resultado de análise de aceitação sensorial, verificou-se que todas as formulações obtiveram índice de aceitação superior a $70 \%$, não havendo diferença significativa entre os resultados de aceitação das formulações testadas $(p<0,05)$. Esses resultados demonstram a eficiência da $\mathrm{MC}$ como barreira a entrada de gordura durante a etapa de pré-fritura, bem como aumento do conteúdo de umidade, tornando-se uma alternativa tecnológica vantajosa para a indústria de alimentos.

Palavras-chave: Produtos empanados. Redução de gordura. Goma metilcelulose.

\begin{abstract}
Even though coated meats have added value to products and gained consumers confidence, by improving these product's appearance and taste, they go through a pre-frying stage during processing which releases the water and allows fat to enter the product, thus increasing fat content. The purpose of this work was to develop a coating system that absorbs less fat during the pre-frying stage of a chicken coated product. To do that, methylcellulose gum was added (MC) in different concentrations in the covering systems, batter (coating liquid) and breading (covering expresses). Five experiments were carried out, a standard experiment, without ingredient addition with technological function, formulations with addition of MC in the batter $(0,5 \%$ and $2,0 \%)$, with addition of $\mathrm{MC}$ in the batter and in the breading $(1 \%$ in each) and with addition of $\mathrm{MC}$ in the breading $(2,0 \%)$. Results showed that fat uptake reduction in formulations with 0,5 and $2,0 \%$ of $\mathrm{MC}$ in the batter were of $5,81 \%$ and $8,40 \%$, respectively, and in experiments with
\end{abstract}

\footnotetext{
1 Doutor em Ciência de Alimentos, Universidade Estadual de Campinas, docente da Universidade do Vale do Rio dos Sinos. Email: luvielmo@unisinos.br. Falta caixa postal.

2 Falta dados sobre $2^{\circ}$. autor
} 
$1 \%$ of $\mathrm{MC}$ in the batter and $1 \%$ of $\mathrm{MC}$ in the breading was of $7,66 \%$. In experiments that received $2 \%$ of MC in the breading, fat uptake reduction was of $10,51 \%$. Formulations with $2 \%$ of MC added to the breading (10,51\%) and $2 \%$ of $\mathrm{MC}$ added to the batter $(8,40 \%)$ presented the largest reductions in fat uptake during the pre-fried stage. Analysis of sensorial acceptance showed that all formulations had a superior acceptance index of $70 \%$, with no significant difference among the other tested formulations acceptance results $(\mathrm{p}<0,05)$. These findings show the efficiency of $\mathrm{MC}$ as a fat uptake barrier during the pre-fried stage, increasing humidity content, and becoming an advantageous technological alternative for the food industry .

Key words: Coated products. Fat-uptake. Methylcellulose gum.

\section{Introdução}

Os sistemas de empanamento tradicionais são compostos de pré-enfarinhamento (predust), líquido ou solução de empanamento (batter) e farinhas de cobertura (farinhas de pão ou rosca, breaders / breadings).

A ordem de adição e utilização destes componentes pode variar, ou seja, não há uma ordem correta e nem sempre são usados todos estes componentes (BORTOLUZZI, 2006; GL，2002; UEMURA; LUZ, 2003).

$\mathrm{O}$ predust consiste em uma farinha fina condimentada ou não, podendo ser constituída de uma combinação de farinhas. O predust é utilizado como camada base (primeira camada) no sistema de cobertura (VIANA, 2005), para pré-condicionar a superfície do produto promovendo a ligação entre o substrato e o batter, absorvendo a umidade da superfície do substrato e assim, melhorar a cobertura, pick-up (rendimento), e adesão/coesão (GL, 2002; BORTOLUZZI, 2006).

Batter é uma mistura em pó de diversos ingredientes funcionais tais como, amidos, gomas e farinhas, podendo ser condimentado ou não. Em processos industriais, ele é preparado em equipamentos específicos, onde é misturado em água, tornando-se uma suspensão de sólidos em líquido pronta para ser aplicada ao produto (GL, 2002; BORTOLUZZI, 2006; LOEWE, 1990).

$O$ batter pode formar a camada de cobertura externa completa para o produto alimentício, como no caso dos sistemas tipo tempura, como também, pode agir como uma camada ligante entre o substrato e a camada mais externa (breading) através de forças físicas e químicas (GL, 2002; CÔRREA, 1997; LOEWE, 1990).

O breading é uma base cereal e geralmente é obtido através de processo térmico, podendo ser condimentado ou não (GL, 2002). A composição do breading pode variar desde farinhas de trigo a misturas com temperos e corantes e são produzidos com granulometria variada e por processos diferentes (DEGENHART, 2003; LOEWE, 1990).

No processo de obtenção dos produtos empanados, a etapa de pré-fritura consiste no mergulho do produto em óleo, sob altas temperaturas $\left(180-200^{\circ} \mathrm{C}\right)$, por um curto período de tempo (20 a $35 \mathrm{~s})$. Esta operação fixa a cobertura, contribui para o desenvolvimento da cor, retira a umidade, inibindo parcialmente da desidratação do produto pelo frio e proporciona absorção de óleo (GL, 2002; DEL RÉ; JORGE, 2006; JORGE; LUNARDI, 2005).

De acordo com Rimac-Brncic et al. (2004), o processo de fritura é largamente utilizado na indústria de alimentos. No entanto, esses produtos, quando fritos, adquirem uma quantia substancial de gordura. Aabsorção de óleo em produtos empanados oscila de $3 \%$ a $60 \%$, podendo ser influenciada por uma série de parâmetros (LAWNSON, 1999; MELLEMA, 2003; JORGE; LUNARDI, 2005).

Neste contexto, alguns hidrocolóides têm sido incorporados às formulações, mostrando sua eficiência como barreira protetora contra a entrada de gorduras nos alimentos (CEREDA; VILPOUX, 2003). Entre estes hidrocolóides, os derivados 
da celulose são os que têm sido estudados mais efetivamente (PENA, 1999).

A metilcelulose apresenta um comportamento incomum, pois possui a capacidade de gelificar-se reversivelmente sob o efeito do calor $\left(50-70^{\circ} \mathrm{C}\right)$, e o gel se rompe após o resfriamento, além de ser solúvel em água fria, insolúvel em gordura, sem qualquer valor calórico, cor e sabor. (PENA, 1999).

$\mathrm{Na}$ indústria de alimentos, umas das mais conhecidas aplicações, em torno da capacidade de formação de gel desta goma é o seu uso nas formulações de batter em base seca (WILLIANS; MITTAL, 1999). Estas gomas são misturadas com outros ingredientes secos, para posteriormente serem hidratados, desempenhando sua função tecnológica (MEYERS, 1990).

Segundo Meyers (1990), esta goma pode ser aplicada aos sistemas de cobertura de três maneiras: a) a primeira é a aplicação direta sobre a superfície do substrato da mesma forma que o predust ou misturada a ele; a segunda é a imersão do substrato em solução contendo goma e água; e a terceira é a aplicação no breading.

Segundo Fizsman e Salvador (2003), quando o produto acrescido de MC é introduzido no óleo quente, ocorre à formação do gel, cria-se uma barreira física à incorporação de óleo e a perda de água, favorecendo uma menor absorção de óleo por parte do produto. Isso é possível em alimentos empanados, quando esta goma é acrescida tanto ao batter quanto ao breading, e serve de barreira à migração de óleo ao produto, bem como impede a saída de água do alimento.

Segundo Roller e Jones (1996), em bolinhos doces fritos do tipo doughnuts, o percentual de gordura reduzido com a utilização de MC na formulação ficou entre $26 \%$ a $28 \%$ sem afetar a aparência e as características sensoriais do produto.

Garcia et al. (2002) estudaram a redução na absorção de gordura, por meio da adição em pó de
1,0\% de MC numa cobertura para bolinhos de batatas, encontrando cerca de 83,6\% de redução de gordura, quando comparado ao controle. Em anéis de lula empanada (tipo tempura), as propriedades de barreira à gordura e umidade foram estudadas em diferentes concentrações e temperaturas por Sanz, Salvador e Fizsman (2004). A redução do conteúdo de gordura foi observada entre $39,0 \%$ e 78,4\% depois da etapa de pré-fritura e $23,1 \%$ e $54,8 \%$ na etapa final de fritura (preparação) usando 1 e 2\% de MC, respectivamente. Nesta mesma concentração, foi possível observar $43,8 \%$ e $75,3 \%$ de umidade retida na etapa de préfritura e $72,2 \%$ e $123,1 \%$ depois da etapa final de fritura, nas mesmas concentrações. Por meio deste estudo, Sanz, Salvador e Fizsman (2004), afirmam que é mais evidente a formação de barreira durante a etapa de pré-fritura, devido à baixa velocidade de absorção de gordura e perda de umidade do batter contendo $\mathrm{MC}$ do que a etapa final de fritura quando o produto é reconstituído e possui menor quantidade de umidade.

Na mesma pesquisa, Sanz, Salvador e Fizsman (2004) comprovaram que o conteúdo de gordura e umidade são influenciados pela temperatura do batter. Em um experimento utilizando 1,5\% de MC adicionada ao batter à temperaturas controladas de $5^{\circ} \mathrm{C}, 15^{\circ} \mathrm{C}$ e $25^{\circ} \mathrm{C}$, pôde ser observada a capacidade de formação de barreira em temperaturas de $5^{\circ} \mathrm{C}$ e a diminuição de barreira conforme o aumento da temperatura.

Com isso verifica-se que baixas temperaturas permitem melhor hidratação da $\mathrm{MC}$, pois desenvolvem maior consistência e propriedades de retenção de água, bem como maior eficiência na geleificação térmica, quando o produto entra em contato com o óleo quente. Sendo assim, o controle da temperatura do batter resultará num melhor desempenho da MC quando adicionada. (SANZ; SALVADOR; FIZSMAN, 2004).

Fizsman e Salvador (2003) apresentam a tabela 1, em que diversos autores comprovaram, por meio de experimentos, a redução de gordura com a utilização de metilcelulose (MC). 
Tabela 1. Estudos utilizando MC nas formulações de diferentes produtos.

\begin{tabular}{lccl}
\hline Referência & MC (\%) & Redução de gordura (\%) & Produto \\
\hline Garcia et al. (2002) & 1,0 & 83,6 & Cobertura para bolinhos de batata*** \\
Fizsman (2004) & $1,0-2,0$ & $23,1-54,8$ & Anéis de lula empanada* \\
Pinthus et al. (1993) & 1,0 & 46,4 & Bolinhos de carne fritos*** \\
Holownia et al. (2000) & 2,0 & 6,5 & Tiras de frango empanadas** \\
\hline
\end{tabular}

* Empanamento tipo tempura.

** Empanamento tradicional (predust, batter, breading).

*** Aplicação direta na massa.

Fonte: Fizsman e Salvador (2003).

Diante da procura dos consumidores por alimentos mais saudáveis, capazes de trazer benefícios à saúde, o presente estudo tem por objetivo reduzir a absorção de gordura durante a etapa de pré-fritura de um produto empanado de frango tipo tradicional (predust, batter e breading), pela adição de metilcelulose em diferentes concentrações nos sistemas de cobertura, batter (líquido de empanamento) e breading (cobertura externa), através da formação de barreira à entrada de gordura.

\section{Material e métodos}

Matéria-prima, ingredientes e processo de fabricação

A matéria-prima utilizada para a elaboração dos empanados foi o recorte de peito de frango resfriado, obtido logo após o corte nas linhas de produção da empresa Doux Frangosul localizada no município de Montenegro - RS.

Os ingredientes utilizados para a elaboração dos empanados foram fornecidos pela empresa Doux Frangosul: água, sal $(\mathrm{NaCl})$, os sistemas de cobertura - predust, batter, breading low salt (baixo teor de sal) do fornecedor GL Laboratories e gordura vegetal de palma, utilizada para fritura do fornecedor Bunge Alimentos. A metilcelulose Methocel A4M foi fornecida pela empresa Dow Chemical Products.

Os empanados foram elaborados (na planta piloto da empresa Doux Frangosul) seguindo as etapas e padrões de processo semelhantes aos adotados em escala industrial (OWENS, 2001; SUDERMAN, 1990).

Os recortes de frango congelados foram moídos em disco de $30 \mathrm{~mm}$, pesados e adicionados os demais ingredientes da massa (água e $\mathrm{NaCl}$ previamente pesados). Foi realizada uma homogeneização da mistura. A massa foi congelada até uma temperatura de -3 a $-4^{\circ} \mathrm{C}$. A massa congelada foi formada em um equipamento específico de moldagem manual. Depois de moldada, a massa recebeu a primeira camada de cobertura - predust e posteriormente o batter. A temperatura do batter manteve-se em torno de $5^{\circ} \mathrm{C}$ e foi padronizado para todos os experimentos 12 segundos de viscosidade medidos no copo Stein. Depois da etapa de cobertura com batter, os experimentos receberam a última etapa de empanamento, breading. Os pick-ups foram avaliados individualmente em cada etapa do processo. Os produtos empanados foram pré-fritos (fritadeira da marca Fry Fritter modelo PR-3000E) em gordura de palma, a temperatura de $185^{\circ} \mathrm{C}$ por 30 segundos. A temperatura do óleo foi medida antes de cada pré-fritura. Os empanados foram cozidos a temperatura de $200^{\circ} \mathrm{C}$ (forno da marca Tedesco modelo Combi TC-6), até a temperatura interna mínima de $80^{\circ} \mathrm{C}$ e congelados individualmente.

A formulação do produto empanado padrão está apresentada na tabela 2 .

A Figura 1 ilustra a aparência do produto após cada etapa do processo de elaboração dos empanados de frango em escala piloto. 
Tabela 2. Formulação padrão do empanado de frango.

\begin{tabular}{lc}
\hline Componentes & $\mathbf{\%}$ \\
\hline Carne & 57,62 \\
Água & 8,98 \\
Sal & 0,40 \\
Predust & 4,00 \\
Batter & 19,00 \\
Breading & 10,00 \\
Total & $\mathbf{1 0 0 , 0 0}$ \\
\hline
\end{tabular}

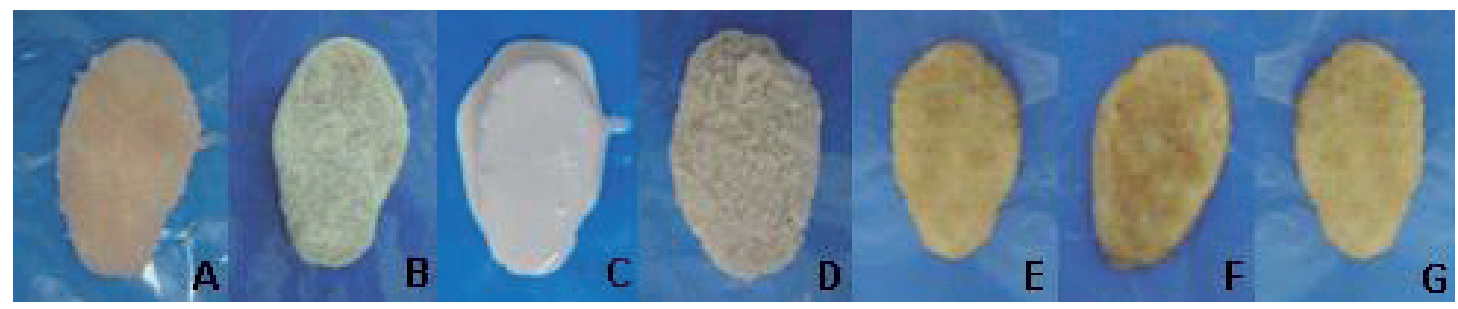

Figura 1. Visual do produto obtido após cada etapa do processo de elaboração do empanado de frango controle (C). A) Massa formada em molde manual (Temperatura da massa: $-3 \mathrm{a}-4^{\circ} \mathrm{C}$ ); B) Aplicação de predust; C) Aplicação de batter; D) Aplicação de breading; E) Produto pré-frito ( $\left.185^{\circ} \mathrm{C} / 30 \mathrm{seg}\right)$; F) Produto cozido $\left(200^{\circ} \mathrm{C} / 5 \mathrm{~min}\right)$; G) Produto Congelado (Temperatura: $-18^{\circ} \mathrm{C}$ ).

\section{Planejamento experimental}

Para verificar o efeito da redução de gordura em produtos empanados de frango através da adição de MC na formulação dos sistemas de cobertura, foram elaborados cinco experimentos com a mesma base cárnea.
A primeira formulação elaborada foi o experimento controle, sem adição de ingrediente com função tecnológica (C). Posteriormente, elaboraram-se dois produtos com adição de metilcelulose no batter $(0,5 \% \mathrm{BA}$ e $2 \% \mathrm{BA})$, um produto com adição de $\mathrm{MC}$ no batter e no breading (1\%BA $1 \%$ BR) e outro com adição de MC no breading ( $2 \% \mathrm{BR})$ (tabela 3$)$.

Tabela 3. Concentrações de MC testadas nos sistemas de cobertura com as respectivas viscosidades.

\begin{tabular}{|c|c|c|c|c|c|}
\hline \multirow{2}{*}{ Legenda } & \multirow{2}{*}{ Formulação } & \multicolumn{2}{|c|}{ Adição de MC } & \multirow{2}{*}{$\begin{array}{c}\text { Diluição } \\
\text { (batter: água gelada) }\end{array}$} & \multirow{2}{*}{$\begin{array}{l}\text { Viscosidade } \\
\text { (s) * }\end{array}$} \\
\hline & & Batter & Breading & & \\
\hline $\mathrm{C}$ & Controle & --- & --- & $1: 1,50$ & 12 \\
\hline $0,5 \% \mathrm{BA}$ & $0,5 \%$ de $\mathrm{MC}$ & $X$ & --- & $1: 1,75$ & 12 \\
\hline $2 \% \mathrm{BA}$ & $2,0 \%$ de $\mathrm{MC}$ & $\mathrm{X}$ & --- & $1: 3,00$ & 12 \\
\hline $1 \%$ BA $1 \%$ BR & $1,0 \%$ de $\mathrm{MC}$ & $\mathrm{X}$ & $X$ & $1: 2,00$ & 12 \\
\hline $2 \% \mathrm{BR}$ & $2,0 \%$ de $\mathrm{MC}$ & --- & $\mathrm{X}$ & $1: 1,50$ & 12 \\
\hline
\end{tabular}

* Copo Stein. 
Para manter as características de aparência e textura do produto, para as quatro diferentes formulações testadas, a viscosidade foi fixada de acordo com o processo de obtenção da formulação controle (12s). Para isso, na etapa do batter, os experimentos receberam diferentes diluições em função da característica tecnológica do ingrediente (aumento de viscosidade). Tanto o tempo de mergulho no batter quanto o tempo de gotejamento foram de aproximadamente $5 \mathrm{~s}$ para garantir o mesmo pick-up de batter nesta etapa do experimento.

\section{Determinações analíticas}

\section{Conteúdo de umidade}

O conteúdo de umidade do produto final foi determinado por método gravimétrico conforme Metodologia do Instituto Adolfo Lutz (1985). A análise foi realizada em triplicata.

Para o cálculo do percentual de umidade retida (UR) durante a etapa de pré-fritura utilizou-se a equação 1 (ALBERT; MITTAL, 2002):

$$
\% U R=\frac{U R_{\text {amostracom } M C}-U R_{\text {amostrasem } M C}}{U R_{\text {amostrasem } M C}} \times 100
$$

\section{Conteúdo de gordura}

O conteúdo de gordura do produto final foi determinado por SOXHLET conforme Metodologia do Instituto Adolfo Lutz (1985), o qual se fundamenta na solubilidade das gorduras em solventes apropriados (éter etílico), seguida da remoção por evaporação ou destilação do solvente empregado. As gorduras extraídas são posteriormente determinadas por gravimetria, após a eliminação do solvente utilizado durante a extração. A análise foi realizada em triplicata.

Para o cálculo do percentual de redução de gordura (RG) durante a etapa de pré-fritura utilizouse a equação 2 (ALBERT; MITTAL, 2002):
$\% R G=\frac{R G_{\text {amostrasemMC }}-R G_{\text {amostracomMC }}}{R G_{\text {amostrasemMC }}} \times 100$

\section{Determinação de viscosidade}

As medições de viscosidade do batter foram realizadas por meio do copo de viscosidade (Copo Stein). O método utilizado de operação do copo de viscosidade está descrito abaixo, sendo a unidade de medida expressa em segundos.

Considerando que o batter foi hidratado nas diluições apresentadas na tabela 4 , foram seguidas as seguintes etapas:

1. Mediu-se a temperatura do batter preparado, em constante agitação.

2. Manteve-se o copo de viscosidade na posição vertical, e mergulhou-se dentro do batter, até que estivesse completamente submerso e cheio.

3. Levantou-se o copo de viscosidade para fora do batter e realizaram-se as seguintes operações de ajuste:

a) o procedimento de tomada de tempo iniciou quando a borda superior do copo de viscosidade surgiu na superfície do batter.

b) o cronômetro foi travado logo que a primeira descontinuidade no fluxo contínuo de batter apareceu, no orifício inferior do copo (e não em qualquer outra parte do fluxo).

\section{Determinação do pick-up}

O pick-up foi controlado por meio da pesagem dos produtos após cada etapa do processo e calculado conforme a equação 3 (GL, 2002):

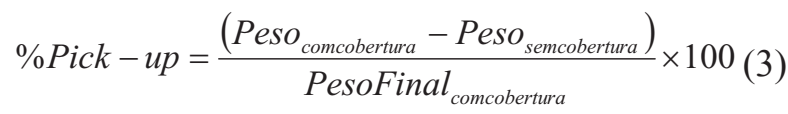




\section{Análise sensorial}

$\mathrm{Na}$ avaliação sensorial dos empanados, foi utilizado o teste de aceitação, com escala hedônica de nove pontos e 30 julgadores não-treinados, conforme metodologia de Teixeira, Meinert e Barbetta (1987).

\section{Análise estatística}

Os cálculos de análise de variância foram realizados utilizando-se o software Statistica 5.0 (STATSOFT, 1999).

\section{Resultados e discussão}

Conteúdo de umidade e gordura

A tabela 4 apresenta os resultados das determinações analíticas de umidade e gordura, por meio da qual foi possível verificar a influência da MC nos experimentos realizados.

Tabela 4. Resultados analíticos das formulações testadas nos sistemas de cobertura.

\begin{tabular}{lcc}
\hline \multicolumn{1}{c}{ Formulação } & $\begin{array}{c}\text { Conteúdo de } \\
\text { Umidade (\%) }\end{array}$ & $\begin{array}{c}\text { Conteúdo de } \\
\text { Gordura (\%) }\end{array}$ \\
\hline Controle & $55,76 \pm 0,85$ & $8,09 \pm 0,35$ \\
$0,5 \%$ de MC & $56,02 \pm 0,70$ & $7,62 \pm 0,73$ \\
$2,0 \%$ de MC & $58,11 \pm 0,77$ & $7,41 \pm 0,48$ \\
$1,0 \%$ de MC & $57,35 \pm 0,62$ & $7,47 \pm 0,89$ \\
$2,0 \%$ de MC & $58,23 \pm 0,75$ & $7,24 \pm 0,51$ \\
\hline
\end{tabular}

Doisfatoresforam considerados nos experimentos que receberam MC nos sistemas de cobertura: o conteúdo de umidade, indicando a retenção de água do produto durante o processo de pré-fritura e o conteúdo de gordura, indicando o teor de redução de gordura durante o processo.
Com base nesses resultados, foi possível calcular através da equação 1 o percentual de redução de gordura e da equação 2 a quantidade de umidade retida na etapa de pré-fritura para as diferentes formulações (tabela 5).

Tabela 5. Resultados de redução de gordura e retenção de umidade durante a pré-fritura.

\begin{tabular}{llcc}
\hline \multicolumn{1}{c}{ Legenda } & Formulação & Redução de Gordura (\%) & $\begin{array}{c}\text { Retenção de Umidade } \\
(\%)\end{array}$ \\
\hline $0,5 \% \mathrm{BA}$ & $0,5 \%$ de $\mathrm{MC}$ & 5,81 & 0,47 \\
$2 \% \mathrm{BA}$ & $2,0 \%$ de $\mathrm{MC}$ & 8,40 & 4,21 \\
$1 \% \mathrm{BA} 1 \% \mathrm{BR}$ & $1,0 \%$ de $\mathrm{MC}$ & 7,66 & 2,85 \\
$2 \% \mathrm{BR}$ & $2,0 \%$ de $\mathrm{MC}$ & 10,51 & 4,43 \\
\hline
\end{tabular}


Para os quatro experimentos, a adição de MC proporcionou redução da absorção de gordura e o aumento da retenção de umidade durante a préfritura dos empanados.

Segundo Dana e Saguy (2006), essa relação acontece, pois, durante o processo de fritura, o óleo é substituído por água e a gordura migra para o produto para tomar o lugar da água que sai. No entanto, se menor quantidade de gordura migrar da fritadeira para o produto, menor será a quantidade de água que migrará do produto para a fritadeira, ou seja, menor será a troca de água por gordura e menor será a absorção.

Com base nos resultados obtidos por Lawnson (1999), verifica-se que, a maioria dos alimentos tendem a acumular maior quantidade de gordura na superfície.

A absorção de óleo em produtos empanados oscila de $3 \%$ a $60 \%$, e pode ser influenciada por uma série de parâmetros, como: alto teor de umidade do produto, relação superfície/volume, sistemas de cobertura, temperatura e qualidade e composição do óleo, os quais atuam sobre as velocidades de transferências de massa e energia entre o óleo e o alimento e que são dependentes do próprio alimento, do óleo e das condições de fritura. $\mathrm{Na}$ maioria dos alimentos, a maior proporção desta gordura absorvida tende a se acumular na superfície do alimento (LAWNSON, 1999).

Holownia et al. (2000) apud Fizsman e Salvador (2003) obtiveram um percentual de redução de gordura de $6,5 \%$ utilizando $2 \%$ de MC num sistema tradicional (predust, batter e breading) aplicado a tiras de frango empanadas. No estudo, não foi mencionado em que componente do sistema de cobertura foi aplicada a goma. Comparando este experimento aos resultados encontrados, verificase um melhor desempenho na aplicação de MC no batter (2\%BA) com 8,40\%, no breading ( $2 \% \mathrm{BR})$ com $10,51 \%$ ou nas duas camadas $(1 \% \mathrm{BA} 1 \% \mathrm{BR})$ com $7,66 \%$.
No experimento $2 \% \mathrm{BR}$, no qual $2 \%$ de $\mathrm{MC}$ foi adicionada ao breading, obtivemos melhor resultado de redução de gordura e maior retenção de umidade, $10,51 \%$ e $4,43 \%$ respectivamente. Quando comparados os experimentos de mesma concentração de MC no produto, percebe-se a maior redução no experimento $2 \% \mathrm{BR}$, seguido do experimento $2 \% \mathrm{BA}$ com $8,40 \%$ de redução de gordura e após com a menor redução de gordura o experimento $1 \% \mathrm{BA} 1 \% \mathrm{BR}$.

Adiferença de redução de gordura do experimento $2 \%$ BR para $2 \%$ BA foi de $2,11 \%$, indicando uma maior eficiência na capacidade de barreira a absorção de gordura, quando utilizamos a MC no breading. Acredita-se que o breading influencie consideravelmente a absorção de gordura durante a pré-fritura em função da alta superfície de contato, e a eficiência na capacidade de barreira a absorção de gordura do batter fique limitada pela utilização do breading.

Segundo Lemos (2003), produtos empanados, sem adição de breading absorvem em torno de $15 \%$ do óleo ou gordura de pré-fritura durante o processo, enquanto os adicionados de breading podem chegar a $20 \%$ de absorção em relação ao peso final do produto. Neste contexto, estes resultados comprovam a influência do breading na absorção de gordura.

Confirmando a influência do breading, verificase o resultado do experimento $1 \% \mathrm{BA} 1 \% \mathrm{BR}$, no qual a $\mathrm{MC}$ foi adicionada tanto no batter quanto no breading, e proporcionou a redução de 7,66\% na gordura intermediária. Supõe-se que ocorreu formação de barreira no batter e também no breading. No entanto, esperava-se um percentual de redução de gordura maior em função do ingrediente ter sido adicionado em ambos os sistemas, porém isso não foi verificado. Atribuem-se a este fato as concentrações de MC serem menores em ambas as camadas $(1 \%)$. 
Determinação de pick-up

O pick-up foi verificado a cada etapa do sistema de cobertura, conforme a equação 3 para os cinco experimentos, garantindo uniformidade dos produtos, bem como a mesma quantidade de ingrediente em todas as camadas de cobertura. $\mathrm{O}$ cuidado maior foi principalmente na camada de batter, à qual a MC foi adicionada. Esse cuidado proporcionou homogeneidade das amostras já que seriam submetidas há determinações analíticas.

Segundo Lemos (2003), a quantidade de cobertura aderida a um produto empanado é um aspecto importante para maior absorção de gordura. Com base nessa afirmação, observa-se na tabela 6 que houve variações na camada externa (pick-up de breading) do experimento $0,5 \% \mathrm{BA}$. Este foi o que apresentou menor redução de gordura ( 5,81\%), e menor retenção de umidade $(0,47 \%)$.

Em função dos valores obtidos (ver tabela 6), é possível que a formulação $0,5 \% \mathrm{BA}$ tenha apresentado um percentual de redução de gordura um pouco menor, por apresentar um pick-up de breading maior. Dessa forma, pode-se verificar que a gordura absorvida no produto aumenta à medida que a quantidade de cobertura aumenta.

\section{Análise sensorial}

Como pode ser observado na tabela 7 , o $\mathrm{F}_{\text {calculado }}$ foi menor que o tabelado, neste caso conclui-se que as formulações não deferem significativamente entre si ao nível de significância de 5\%.

Tabela 6. Valores médios de pick-up obtidos durante a elaboração dos empanados.

\begin{tabular}{cccccc}
\hline $\begin{array}{c}\text { Camada de } \\
\text { cobertura }\end{array}$ & $\begin{array}{c}\text { Controle } \\
(\mathbf{C})\end{array}$ & $\begin{array}{c}\mathbf{0 , 5 \%} \text { de MC } \\
\mathbf{( 0 , 5 \% B A )}\end{array}$ & $\begin{array}{c}\mathbf{2 , 0 \%} \text { de MC } \\
\mathbf{( 2 \% B A )}\end{array}$ & $\begin{array}{c}\mathbf{1 , 0 \%} \text { de MC } \\
(\mathbf{1 \% B A 1 \% B R})\end{array}$ & $\begin{array}{c}\mathbf{2 , 0 \%} \text { de MC } \\
(\mathbf{2 \%} \% \mathbf{B R})\end{array}$ \\
\hline Predust & 4,0 & 3,7 & 3,1 & 4,9 & 4,3 \\
Batter & 19,0 & 18,5 & 18,9 & 17,0 & 18,8 \\
Breading & 10,0 & 11,5 & 10,4 & 9,5 & 10,5 \\
\hline
\end{tabular}

Tabela 7. Resultado da análise de variância

\begin{tabular}{cccccc}
\hline $\mathbf{C V}$ & $\mathbf{G L}$ & $\mathbf{S . Q .}$ & $\mathbf{Q . M .}$ & $\mathbf{F}_{\text {calc }}$ & $\mathbf{F}_{\text {tab. }}$ \\
\hline Formulação & 3 & 1,4 & 0,4667 & 0,37214 & 2,724 \\
Julgadores & 29 & 64 & 2,2172 & 1,76810 & 1,614 \\
Resíduo & 87 & 109,1 & 1,2502 & --- & --- \\
Total & 119 & 174,8 & --- & --- & --- \\
\hline
\end{tabular}




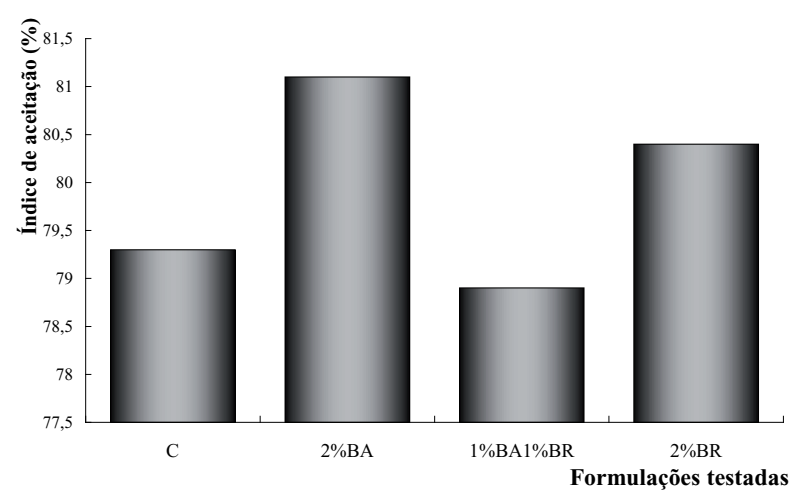

Figura 2. Índice de Aceitação das formulações testadas sensorialmente.

Como pode ser observado na Figura 2, as médias de aceitação dos quatro experimentos que apresentaram melhores percentuais de redução de gordura ficaram acima de $70 \%$. Isso significa que todas as amostras foram aceitas entre os julgadores, ficando estes valores entre o "gostei ligeiramente" e o "gostei muito".

Segundo Roller e Jones (1996), a adição de MC na formulação não afeta a aparência e as características sensoriais do produto, no estudo realizado por Roller e Jones (1996) os julgadores não encontraram diferença significativa entre as formulações testadas. Isso é bastante interessante, ao passo que a adição de um ingrediente tecnológico, além de manter as características iniciais do produto, proporciona benefícios relacionados à redução de gorduras tornando os produtos mais saudáveis.

\section{Conclusão}

A utilização da MC nas formulações de produtos empanados de frango, não alterou as características sensoriais do produto. As formulações com $2 \%$ de $\mathrm{MC}$ adicionada ao breading e $2 \%$ de $\mathrm{MC}$ adicionada ao batter obtiveram as maiores reduções de absorção de gordura durante a etapa de pré-fritura, $10,51 \%$ e $8,40 \%$ respectivamente. As formulações com $2 \%$ de MC adicionada ao breading e 2\% de MC adicionada ao batter obtiveram as maiores retenções de água no produto, $4,21 \%$ e $4,43 \%$, respectivamente.
Estes resultados demonstram a eficiência da barreira de MC adicionada ao empanamento, na troca água-gordura durante a pré-fritura, tornando vantajosa a utilização industrial deste ingrediente para redução de gordura em produtos empanados.

\section{Referências}

ALBERT, S.; MITTAL, G. S. Comparative evaluation of edible coatings to reduce fat uptake in a deep-fried cereal product. Food research International, Barking, v. 35, n. 5,p. 445-458, 2002.

BORTOLUZZI, R. C. Empanados. In: OLIVO, R. (Ed.). $O$ mundo do frango: cadeia produtiva da carne de frango. Criciúma: Ed. do Autor, 2006. p. 481-494.

CEREDA, M. P.; VILPOUX, O. F. O amido com aplicação em produtos para alimentação saudável. In: . (Org.). Tecnologia, usos e potencialidades de tuberosas amiláceas Latino Americanas. São Paulo: Fundação Cargill, 2003. v. 3, p. 355-376.

CORRÊA, M. S. Tecnologia de empanados. Revista Nacional da Carne, São Paulo, n. 249, p. 34-40, nov. 1997.

DANA, D.; SAGUY, S. I. Rewiew: Mechanism of oil uptake during deep-fat frying and the surfactant effecttheory and myth. Advances in Colloid and Interface Science, Amsterdam, v. 130, n. 2, p. 267-272, 2006.

DEGENHARDT, J. Empanamento de produtos cárneos. Aditivos \& Ingredientes, São Paulo, n. 28, p. 77-79, set./ dez. 2003.

DEL RÉ, P. V.; JORGE, N. Comportamento de óleos vegetais em frituras descontínuas de produtos préfritos congelados. Ciência e Tecnologia de Alimentos. Campinas, v. 26, n. 1, p.56-63, 2006.

FIZSMAN, S. M.; SALVADOR, A. Recent Development in coating batters. Trends in Food Science \& Technology Cambridge, v. 14, n. 10, p. 399-407, 2003.

GARCIA, M. A; FERRERO, C.; BÉRTOLA, N.; MARTINO, M.; ZARITZKI, N. Edible coatings from celullose derivatives to reduce oil uptake in fried foods. Innovative Food Science and Emerging Technologies, New York, v.3, n. 4, p. 391-397, 2002.

GL-Laboratories worldwide. Guia completo para sistemas de cobertura. Guarulhos: GLLW, 2002. 41 p.

INSTITUTO ADOLFO LUTZ. Normas analíticas do Instituto Adolfo Lutz. 3.ed. São Paulo: Governo do estado de São Paulo, Secretaria dos serviços da saúde, 1985. v. 1 . 
JORGE, N.; LUNARDI, M. V. Influência dos tipos de óleo e tempos de fritura na perda de umidade e absorção de óleo em batatas fritas. Ciências Agrotécnicas, Lavras, v. 29 , n. 3, p. 635-641, 2005.

LAWNSON, H. Aceites y grasas alimentarios: tecnologia, utilización y nutrición. Zaragoza: Acribia, 1999.

LEMOS, A. L. S. C. Empanamento: valor agregado e conveniência para produtos cárneos. In: .Tópicos especiais: processamento da carne de aves. Campinas: CTC/ITAL, 2003. v. 1, p. 112-114.

LOEWE, R. Ingredients selection for batters systems. In: KULP, K.; LOEWE, R. (Ed.). Batters and breadings in food procesing. Minnesota: American Association of Cereal Chemists, 1990. p. 11-28.

MELLEMA, M. Mechanism and reduction of fat uptake in deep-fat fried foods. Trends in Food Science \& Technology, Cambridge, v. 14, n. 9, p. 364-373, 2003.

MEYERS, M. A. Functionality of hydrocolloids in batter coating systems. In: KULP, K.; LOEWE, R. (Ed.). Batters and Breadings in Food Processing St. Paul. Minnesota: American Association of Cereal Chemists, 1990. p.117-141.

OWENS, C. M. Coated poultry products. In: SAMS, A. R. Poultry Meat Processing. Boca Raton: Lewis Publishers, 2001. p. 227-242.

PENA, A. L. Hidrocolóides: uso em alimentos. Food Ingredients, São Paulo, v. 1, p. 31-35, 1999.
RIMAC-BRNCIC， S.; LELAS， V.; RADE， D.; SIMUNDIC, B. Decreasing of oil absortion in potato strips during deep fat frying. Journal of Food Engineering, Essex, v. 64, n. 2, p. 237-241, 2004.

ROLLER, S.; JONES, S. A. Handbook of fat replacers. Boca Raton: Crc, 1996.

SANZ, T.; SALVADOR, A.; FIZSMAN, S. M. Effect of concentration and temperature on properties of methylcellulose-added batters: application to battered, fried seafood. Food Hidrocolloids, New York, v. 18, n. 1, p. 127-131, 2004.

STATSOFT Incorporation. Statistica 5.0. Tuse, 1999.

SUDERMAN, R. D. Applications of Batters and Breadings to Poultry, Seafood, Red Meat and Vegetables. In: KULP, K; LOEWE, R. (Ed.). Batters and breadings in food processing. Minnesota: American Association of Cereal Chemists, 1990. p. 275-277.

TEIXEIRA, E.; MEINERT, E. M.; BARBETTA, P. A. Análise sensorial de alimentos. Florianópolis: Universidade Federal de Santa Catarina, 1987.

UEMURA, C. H.; LUZ, M. B. Sistemas de cobertura. Aditivos \& Ingredientes, São Paulo, n. 28, p. 81-82, set/ dez., 2003.

\section{VIANA, 2005 - Não existe referência}

WILLIAMS, R.; MITTAL, G. S. Water and fat transfer properties of polysaccharide films on fried pastry mix. Lebensmittel-Wissenschaft und-Technologie, London, $\mathrm{v}$ 32, n. 7, p. 440-445, 1999. 
